\title{
Multipoint Approximation of Statistical Descriptors of Local Strain and Stress Fields in Heterogeneous Media Using Integral Equation Method
}

\author{
Mikhail Tashkinov 1 \\ Faculty of Applied Mathematics and Mechanics, Perm National Research Polytechnic University, \\ Komsomolsky Ave. 29, Perm 614990, Russia \\ Correspondence should be addressed to Mikhail Tashkinov; m.tashkinov@pstu.ru
}

Received 30 July 2017; Accepted 28 March 2018; Published 6 May 2018

Academic Editor: Ricardo Weder

Copyright (C) 2018 Mikhail Tashkinov. This is an open access article distributed under the Creative Commons Attribution License, which permits unrestricted use, distribution, and reproduction in any medium, provided the original work is properly cited.

\begin{abstract}
This paper is devoted to derivation of analytic expressions for statistical descriptors of stress and strain fields in heterogeneous media. Multipoint approximations of solutions of stochastic elastic boundary value problems for representative volume elements are investigated. The stress and strain fields are represented in the form of random coordinate functions, for which analytical expressions for the first- and second-order statistical central moments are obtained. Such moments characterize distribution of fields under prescribed loading of a representative volume element and depend on the geometry features and location of components within a volume. The information of the internal geometrical structure is taken into account by means of multipoint correlation functions. Within the framework of the second approximation of the boundary value problem, the correlation functions up to the fifth order are required to calculate the statistical characteristics. Using the method of Green's functions, analytical expressions for the moments in distinct phases of the microstructure are obtained explicitly in a form of integral equations. Their analysis and comparison with previously obtained results are performed.
\end{abstract}

\section{Introduction}

Heterogeneous materials nowadays are the essential link of advanced engineering solutions. The advantages of such materials are underpinned by an ability to obtain tailored properties by combining constituents and their characteristics on microscale level. In the context of solving problems connected with evaluation of microstructural behavior of such heterogeneous materials and media, it is necessary to take into account mutual influence of inhomogeneities. As a rule, modeling of the effective response of materials is performed within the concept of representative volume element (RVE). In these frameworks, wide range of mechanical approaches can be used to establish connection between different scales of materials with complex microstructure. Randomly reinforced media can be distinguished in a separate class of materials where local mechanical characteristics can be estimated using statistical instruments and the theory of random functions. Thus, moments of the stress and strain fields in components can be used as the statistical measures of local mechanical behavior [1]. The morphological properties of the microstructure can be formalized using correlation functions that describe interaction of the inhomogeneities with each other and can sense geometrical effects, such as shape, distribution, clustering, and percolation [2].

Statistical assessment of mechanical behavior can be done analytically for each phase of media. Kroener [3] and Beran [4] developed statistical mathematical formulations in order to connect geometrical correlation functions and properties of heterogeneous materials. Explicit relations for second moments of stresses were obtained in [5] and utilized second- and third-order interactions between inclusions. Several exact solutions for second-order moment of stress fields were offered for some cases of deterministic structures. One of such models is media with regular structure [6-8]. The method of integral equations can be implemented for obtaining analytical expressions for local statistics. It presumes that mechanical properties of microstructural components 
are defined with conventional phenomenological equations and criteria while microscopic strain and stress fields are computed using the solutions of stochastic boundary value problems (SBVPs) with rapidly oscillating coefficients. The numerous ways of resolving and closing the integral equations were offered [9-21].

The commonly applied simplification when constructing statistical metrics for heterogeneous RVEs is that only lower order interaction between the microstructural elements is being considered. This work contains explicit derivation of the expressions for local statistical descriptors of stress and strain in heterogeneous media using multipoint high order approximation of SBVP solution and second derivative expansion of Green's function approach.

One of the ground concepts used in statistical analysis of random structures and also applied in this work is based on perturbation approach $[4,16,22,23]$ and presumes that local stress and strain fields as well as local stiffness tensor depend on position of the radius vector and can be decomposed into an averaged value and random addition:

$$
\begin{aligned}
\sigma_{i j}(\vec{r}) & =\left\langle\sigma_{i j}(\vec{r})\right\rangle+\sigma_{i j}^{\prime}(\vec{r}) \\
\varepsilon_{i j}(\vec{r}) & =\left\langle\varepsilon_{i j}(\vec{r})\right\rangle+\varepsilon^{\prime}{ }_{i j}(\vec{r}) \\
C_{i j k l}(\vec{r}) & =\left\langle C_{i j k l}(\vec{r})\right\rangle+C_{i j k l}^{\prime}(\vec{r}),
\end{aligned}
$$

where $\left\langle\sigma^{\prime}{ }_{i j}(\vec{r})\right\rangle=\left\langle\varepsilon^{\prime}{ }_{i j}(\vec{r})\right\rangle=\left\langle C^{\prime}{ }_{i j k l}(\vec{r})\right\rangle=0, \vec{r}$ is radius vector with components $\left(x_{1}, x_{2}, x_{3}\right)$. The averaging procedure for any coordinate-dependent value $f(\vec{r})$ is determined as

$$
\langle f(\vec{r})\rangle=\frac{1}{V} \int_{V} f(\vec{r}) d V
$$

where integration is performed over the representative volume $V$.

\section{Approximation of Stochastic Boundary Value Problem Solution}

Stochastic boundary value problem (SBVP) of elasticity theory for RVEs of heterogeneous materials with random microstructural elements can be defined with the following equations:

$$
\begin{aligned}
\sigma_{i j, j}(\vec{r}) & =0 \\
\varepsilon_{i j}(\vec{r}) & =\frac{1}{2}\left(u_{i, j}(\vec{r})+u_{j, i}(\vec{r})\right) \\
\sigma_{i j}(\vec{r}) & =C_{i j k l}(\vec{r}) \varepsilon_{k l}(\vec{r}) \\
\left.u_{i}(\vec{r})\right|_{\vec{r} \in \Gamma_{u}} & =e_{i j} r_{j},
\end{aligned}
$$

where $C_{i j k l}(\vec{r})$ is stiffness tensor, $e_{i j}$ are components of tensor of small strains which define applied load, and $r_{j}$ are coordinates of the surface points. In this formulation it is assumed that conditions of ideal contact are satisfied on the interphase surface. The boundary value problem is statistically nonlinear since its physical equations (5) contain multiplication of random fields.

Due to ergodicity hypothesis volume averaging is equal to statistical averaging: $\varepsilon_{i j}^{*}=\left\langle\varepsilon_{i j}(\vec{r})\right\rangle, \sigma_{i j}^{*}=\left\langle\sigma_{i j}(\vec{r})\right\rangle$.

For two-phase heterogeneous media, containing matrix and one type of inclusions, the stiffness tensor $C_{i j k l}(\vec{r})$ is defined as

$$
C_{i j k l}(\vec{r})=\lambda(\vec{r}) C_{i j k l}^{I}+(1-\lambda(\vec{r})) C_{i j k l}^{M},
$$

where $C_{i j k l}^{I}$ and $C_{i j k l}^{M}$ are constant stiffness tensors of inclusions and matrix, respectively. The indicator function $\lambda(\vec{r})$ is defined as

$$
\lambda(\vec{r})= \begin{cases}1, & \vec{r} \in V_{I} \\ 0, & \vec{r} \in V_{M},\end{cases}
$$

where $V_{I}$ is spatial volume of inclusions and $V_{M}$ is volume of matrix.

After averaging (7), the constant tensor, depending on volume fraction, is obtained:

$$
\left\langle C_{i j k l}(\vec{r})\right\rangle=p C_{i j k l}^{I}+(1-p) C_{i j k l}^{M},
$$

where $p=\langle\lambda(\vec{r})\rangle$ is volume fraction of inclusions.

Going back to solution of the boundary value problem, (3)-(6) can be transformed into the following representation:

$$
\left(C_{i j k l}(\vec{r}) \frac{1}{2}\left(u_{k, l}(\vec{r})+u_{l, k}(\vec{r})\right)\right)_{, j}=0
$$

Due to symmetrical properties of the stiffness tensor $C_{i j k l}(\vec{r})=C_{i j l k}(\vec{r})$ it can be reduced to

$$
\left(C_{i j k l}(\vec{r}) u_{k, l}(\vec{r})\right)_{, j}=0 .
$$

Applying the perturbation approach, (11) can be expressed through mean and fluctuation:

$$
\begin{aligned}
& \left(\left(\left\langle C_{i j k l}\right\rangle+C_{i j k l}^{\prime}(\vec{r})\right)\left(\left\langle u_{k}(\vec{r})\right\rangle_{, l}+u_{k, l}^{\prime}(\vec{r})\right)\right)_{, j} \\
& \quad=0 .
\end{aligned}
$$

Here, averaged values $\left\langle C_{i j k l}\right\rangle$ and $\left\langle u_{k}(\vec{r})\right\rangle$ are expressed via (9) and boundary conditions (6).

Expanding (12) gives the following:

$$
\begin{aligned}
& \left(\left\langle C_{i j k l}\right\rangle u_{k, l}^{\prime}(\vec{r})\right)_{, j} \\
& =-\left(C_{i j k l}^{\prime}(\vec{r}) e_{k l}+C_{i j k l}^{\prime}(\vec{r}) u_{k, l}^{\prime}(\vec{r})\right)_{, j} .
\end{aligned}
$$

Denoting the right part as $P_{i j, j}(\vec{r})$ :

$$
\left\langle C_{i j k l}\right\rangle u_{k, l j}^{\prime}(\vec{r})=-P_{i j, j}(\vec{r}) .
$$

Solution of this differential equation in displacements $u_{k}^{\prime}(\vec{r})$ can be found using Green's function method. According to definition of Green's functions [24], for the constant 
tensor $\left\langle C_{i j k l}\right\rangle$ Green's function $G_{k n}\left(\vec{r}, \vec{r}_{1}\right)$ and its derivatives are equal 0 in infinity and satisfy

$$
\left\langle C_{i j k l}\right\rangle G_{k n, j l}\left(\vec{r}, \vec{r}_{1}\right)=-\delta_{i n} \delta\left(\vec{r}-\vec{r}_{1}\right) .
$$

Here, $\delta\left(\vec{r}-\vec{r}_{1}\right)$ is Dirac's delta function and $\delta_{i n}$ is Kronecker symbol.

Using Green's function, fluctuations of displacements $u_{i}^{\prime}(\vec{r})$ are obtained as the following integral equation:

$$
u_{i}^{\prime}(\vec{r})=\int_{V_{1}} G_{i j}\left(\vec{r}, \vec{r}_{1}\right) \frac{\partial P_{j n}\left(\vec{r}_{1}\right)}{\partial x_{1 n}} d V_{1}
$$

Such representation of fluctuations of the local displacement field can be used in expressions for statistical descriptors of random fields-multipoint moments of various orders.

\section{Statistical Descriptors of Local Stress and Strain Fields}

In the general case of a multicomponent material, an indicator function $\lambda_{C}(\vec{r})$ takes the value 1 if the radius vector is in component $C$ and is equal to 0 in all other cases. Then the local statistical moments of the first and second order in the component $C$ of heterogeneous media are determined with the following relations [20]:

$$
\begin{aligned}
& \left\langle\varepsilon_{i j}\right\rangle_{C}=e_{i j}+\frac{1}{\left\langle\lambda_{C}\right\rangle}\left\langle\lambda_{C}^{\prime}(\vec{r}) \varepsilon_{i j}^{\prime}(\vec{r})\right\rangle \\
& \left\langle\sigma_{i j}\right\rangle_{C}=\left\langle\sigma_{i j}\right\rangle+\frac{1}{\left\langle\lambda_{C}\right\rangle}\left\langle\lambda_{C}^{\prime}(\vec{r}) \sigma_{i j}^{\prime}(\vec{r})\right\rangle \\
& \left\langle\varepsilon_{i j}^{\prime}(\vec{r}) \varepsilon_{\alpha \beta}^{\prime}(\vec{r})\right\rangle_{C}=\left\langle\varepsilon_{i j}^{\prime}(\vec{r}) \varepsilon_{\alpha \beta}^{\prime}(\vec{r})\right\rangle+e_{i j} e_{\alpha \beta} \\
& -\left\langle\varepsilon_{i j}\right\rangle_{C}\left\langle\varepsilon_{\alpha \beta}\right\rangle_{C} \\
& +\frac{1}{\left\langle\lambda_{C}\right\rangle}\left(\left\langle\lambda_{C}^{\prime}(\vec{r}) \varepsilon_{i j}^{\prime}(\vec{r}) \varepsilon_{\alpha \beta}^{\prime}(\vec{r})\right\rangle\right. \\
& +e_{i j}\left\langle\lambda_{C}^{\prime}(\vec{r}) \varepsilon^{\prime}{ }_{\alpha \beta}(\vec{r})\right\rangle \\
& \left.+e_{\alpha \beta}\left\langle\lambda_{C}^{\prime}(\vec{r}) \varepsilon_{i j}^{\prime}{ }_{i j}(\vec{r})\right\rangle\right) \\
& \left\langle\sigma_{i j}^{\prime}(\vec{r}) \sigma_{\alpha \beta}^{\prime}(\vec{r})\right\rangle_{C}=\left\langle\sigma_{i j}^{\prime}(\vec{r}) \sigma_{\alpha \beta}^{\prime}(\vec{r})\right\rangle+\left\langle\sigma_{i j}\right\rangle \\
& +\left\langle\sigma_{\alpha \beta}\right\rangle-\left\langle\sigma_{i j}\right\rangle_{C}\left\langle\sigma_{\alpha \beta}\right\rangle_{C} \\
& +\frac{1}{\left\langle\lambda_{C}\right\rangle}\left(\left\langle\lambda_{C}^{\prime}(\vec{r}) \sigma_{i j}^{\prime}(\vec{r}) \sigma_{\alpha \beta}^{\prime}(\vec{r})\right\rangle\right. \\
& +\left\langle\sigma_{i j}\right\rangle\left\langle\lambda_{C}^{\prime}(\vec{r}) \sigma_{\alpha \beta}^{\prime}(\vec{r})\right\rangle \\
& \left.+\left\langle\sigma_{\alpha \beta}\right\rangle\left\langle\lambda_{C}^{\prime}(\vec{r}) \sigma_{i j}^{\prime}(\vec{r})\right\rangle\right) .
\end{aligned}
$$

Random fields of fluctuations of stresses and strains with regard to the statement of the boundary value problem are defined as

$$
\begin{aligned}
\varepsilon_{i j}^{\prime}(\vec{r})= & \frac{1}{2}\left(u_{i, j}^{\prime}(\vec{r})+u_{j, i}^{\prime}(\vec{r})\right) \\
\sigma_{i j}^{\prime}(\vec{r})= & \sigma_{i j}(\vec{r})-\left\langle\sigma_{i j}\right\rangle \\
= & C^{\prime}{ }_{i j k l}(\vec{r}) e_{k l}-\left\langle C_{i j k l}^{\prime}(\vec{r}) \varepsilon^{\prime}{ }_{k l}(\vec{r})\right\rangle \\
& +C_{i j k l}(\vec{r}) \varepsilon^{\prime}{ }_{k l}(\vec{r}) .
\end{aligned}
$$

Thus, the moments for the unknown statistical characteristics depend on the fluctuations of the field of the indicator function $\lambda_{C}^{\prime}(\vec{r})$, the displacements $u_{i}^{\prime}(\vec{r})$, and strains $\varepsilon_{i j}^{\prime}(\vec{r})$.

Returning to two-phase materials, taking into account the latest transformations, the moments containing stress fluctuations can be expressed in terms of the moments containing strain fluctuations:

$$
\begin{aligned}
& \left\langle\sigma_{i j}^{\prime}(\vec{r}) \sigma_{\alpha \beta}^{\prime}(\vec{r})\right\rangle=e_{k l} e_{\phi h} \bar{C}_{i j k l} \bar{C}_{\alpha \beta \phi h} D_{\lambda}^{(2)} \\
& +e_{k l} \bar{C}_{i j k l}\left\langle C_{\alpha \beta \phi h}\right\rangle\left\langle\lambda^{\prime}(\vec{r}) \varepsilon^{\prime}{ }_{\phi h}(\vec{r})\right\rangle \\
& +e_{\phi h} \bar{C}_{\alpha \beta \phi h}\left\langle C_{i j k l}\right\rangle\left\langle\lambda^{\prime}(\vec{r}) \varepsilon^{\prime}{ }_{k l}(\vec{r})\right\rangle+\left\langle C_{i j k l}\right\rangle \\
& \cdot\left\langle C_{\alpha \beta \phi h}\right\rangle\left\langle\varepsilon^{\prime}{ }_{\phi h}(\vec{r}) \varepsilon^{\prime}{ }_{k l}(\vec{r})\right\rangle-e_{k l} \bar{C}_{i j k l} \bar{C}_{\alpha \beta \phi h}(1 \\
& -2 p)\left\langle\lambda^{\prime}(\vec{r}) \varepsilon_{\phi h}^{\prime}(\vec{r})\right\rangle-e_{\phi h} \bar{C}_{i j k l} \bar{C}_{\alpha \beta \phi h}(1-2 p) \\
& \cdot\left\langle\lambda^{\prime}(\vec{r}) \varepsilon^{\prime}{ }_{k l}(\vec{r})\right\rangle-\bar{C}_{\alpha \beta \phi h}\left\langle C_{i j k l}\right\rangle\left\langle\lambda^{\prime}(\vec{r})\right. \\
& \left.\cdot \varepsilon^{\prime}{ }_{\phi h}(\vec{r}) \varepsilon_{k l}^{\prime}(\vec{r})\right\rangle-\bar{C}_{i j k l}\left\langle C_{\alpha \beta \phi h}\right\rangle\left\langle\lambda^{\prime}(\vec{r})\right. \\
& \left.\cdot \varepsilon^{\prime}{ }_{\phi h}(\vec{r}) \varepsilon^{\prime}{ }_{k l}(\vec{r})\right\rangle+\bar{C}_{i j k l} \bar{C}_{\alpha \beta \phi h}((1-2 p) \\
& \cdot\left\langle\lambda^{\prime}(\vec{r}) \varepsilon_{k l}^{\prime}(\vec{r}) \varepsilon_{\phi h}^{\prime}(\vec{r})\right\rangle \\
& \left.+D_{\lambda}^{(2)}\left\langle\varepsilon^{\prime}{ }_{k l}(\vec{r}) \varepsilon_{\phi h}^{\prime}(\vec{r})\right\rangle\right) \\
& \left\langle\lambda^{\prime}(\vec{r}) \sigma_{i j}^{\prime}(\vec{r})\right\rangle=e_{k l} \bar{C}_{i j k l} D_{\lambda}^{(2)}-\bar{C}_{i j k l}(1-2 p) \\
& \cdot\left\langle\lambda^{\prime}(\vec{r}) \varepsilon^{\prime}{ }_{k l}(\vec{r})\right\rangle+\left\langle C_{i j k l}\right\rangle\left\langle\lambda^{\prime}(\vec{r}) \varepsilon^{\prime}{ }_{k l}(\vec{r})\right\rangle \\
& \left\langle\lambda^{\prime}(\vec{r}) \sigma_{i j}^{\prime}(\vec{r}) \sigma_{\alpha \beta}^{\prime}(\vec{r})\right\rangle=e_{k l} e_{\phi h} \bar{C}_{i j k l} \bar{C}_{\alpha \beta \phi h} D_{\lambda}^{(3)} \\
& +e_{k l} \bar{C}_{i j k l}(1-2 p)\left\langle C_{\alpha \beta \phi h}\right\rangle\left\langle\lambda^{\prime}(\vec{r}) \varepsilon^{\prime}{ }_{\phi h}(\vec{r})\right\rangle \\
& +e_{\phi h} \bar{C}_{\alpha \beta \phi h}(1-2 p)\left\langle C_{i j k l}\right\rangle\left\langle\lambda^{\prime}(\vec{r}) \varepsilon^{\prime}{ }_{k l}(\vec{r})\right\rangle \\
& +\left\langle C_{i j k l}\right\rangle\left\langle C_{\alpha \beta \phi h}\right\rangle\left\langle\lambda^{\prime}(\vec{r}) \varepsilon^{\prime}{ }_{\phi h}(\vec{r}) \varepsilon^{\prime}{ }_{k l}(\vec{r})\right\rangle \\
& -e_{k l} \bar{C}_{i j k l} \bar{C}_{\alpha \beta \phi h}\left(1-3 D_{\lambda}^{(2)}\right)\left\langle\lambda^{\prime}(\vec{r}) \varepsilon_{\phi h}^{\prime}(\vec{r})\right\rangle \\
& -e_{\phi h} \bar{C}_{i j k l} \bar{C}_{\alpha \beta \phi h}\left(1-3 D_{\lambda}^{(2)}\right)\left\langle\lambda^{\prime}(\vec{r}) \varepsilon^{\prime}{ }_{k l}(\vec{r})\right\rangle \\
& -\left(\bar{C}_{\alpha \beta \phi h}\left\langle C_{i j k l}\right\rangle+\bar{C}_{i j k l}\left\langle C_{\alpha \beta \phi h}\right\rangle\right)((1-2 p) \\
& \cdot\left\langle\lambda^{\prime}(\vec{r}) \varepsilon_{k l}^{\prime}(\vec{r}) \varepsilon_{\phi h}^{\prime}(\vec{r})\right\rangle
\end{aligned}
$$




$$
\begin{aligned}
& \left.+D_{\lambda}^{(2)}\left\langle\varepsilon^{\prime}{ }_{k l}(\vec{r}) \varepsilon^{\prime}{ }_{\phi h}(\vec{r})\right\rangle\right) \\
& +\bar{C}_{i j k l} \bar{C}_{\alpha \beta \phi h}\left(\left(1-3 D_{\lambda}^{(2)}\right)\right. \\
& \cdot\left\langle\lambda^{\prime}(\vec{r}) \varepsilon^{\prime}{ }_{k l}(\vec{r}) \varepsilon^{\prime}{ }_{\phi h}(\vec{r})\right\rangle \\
& \left.+D_{\lambda}^{(3)}\left\langle\varepsilon^{\prime}{ }_{k l}(\vec{r}) \varepsilon^{\prime}{ }_{\phi h}(\vec{r})\right\rangle\right) .
\end{aligned}
$$

Here, the tensor $\bar{C}_{i j k l}=C_{i j k l}^{I}-C_{i j k l}^{M}$ is the difference of the stiffness tensors of the inclusions and the matrix.

In order to calculate the local moments of the strain and stress fields, it is necessary to know the analytical form of the moment $\left\langle\varepsilon^{\prime}{ }_{k l}(\vec{r}) \varepsilon^{\prime}{ }_{\phi h}(\vec{r})\right\rangle$ and also mixed unconditional moments $\left\langle\lambda^{\prime}(\vec{r}) \varepsilon^{\prime}{ }_{k l}(\vec{r})\right\rangle$ and $\left\langle\lambda^{\prime}(\vec{r}) \varepsilon^{\prime}{ }_{\phi h}(\vec{r}) \varepsilon^{\prime}{ }_{k l}(\vec{r})\right\rangle$. These moments are not related to certain phase and characterize the RVE as homogeneous medium. All moments necessary for calculating the statistical characteristics depend on the geometry of the microstructure, its physical properties, and loading conditions. The physical properties of the components of the structure are determined by the tensors of the moduli of elasticity of inclusions and the matrix $C_{i j k l}^{I}$, $C_{i j k l}^{M}$. The geometry of the structure is characterized by the volume fraction $p$, the central moments of the indicator function $D_{\lambda}^{(n)}$, and multipoint high order correlation functions.

\section{Integral Equations for Statistical Descriptors}

After differentiating and expanding, expression (16) can be rewritten as

$$
\begin{aligned}
& u_{i, j}^{\prime}(\vec{r})=\int_{V_{1}} G_{i m, j}\left(\vec{r}, \vec{r}_{1}\right)\left(C_{m n k l}^{\prime}\left(\vec{r}_{1}\right) e_{k l}\right. \\
& \left.+C_{m n k l}^{\prime}\left(\vec{r}_{1}\right) u_{k, l}^{\prime}\left(\vec{r}_{1}\right)\right)_{, 1 n} d V_{1} .
\end{aligned}
$$

The displacement fluctuations $u^{\prime}(\vec{r})$, with respect to which the equation is being solved, are both on the left and on the right side of the expression, so the successive approximations are used for the solution. The recurrence sequence has the following form:

$$
\begin{aligned}
& u_{i, j}^{\prime(\chi)}(\vec{r})=\int_{V_{1}} G_{i m, j}\left(\vec{r}, \vec{r}_{1}\right)\left(C_{m n k l}^{\prime}\left(\vec{r}_{1}\right) e_{k l}\right. \\
& \left.+C_{m n k l}^{\prime}\left(\vec{r}_{1}\right) u_{k, l}^{\prime(\chi-1)}\left(\vec{r}_{1}\right)\right)_{, 1 n} d V_{1}
\end{aligned}
$$

where $\chi$ is order of approximation.

This paper utilizes the second approximation, which contributes to the accuracy of the solution by taking into account multipoint structural correlation functions:

$$
\begin{aligned}
& u_{i, j}^{\prime(2)}(\vec{r})=e_{k l} \int_{V_{1}} G_{i m, j}\left(\vec{r}, \vec{r}_{1}\right) \\
& \cdot\left(C_{m n k l}^{\prime}\left(\vec{r}_{1}\right)\right)_{, 1 n} d V_{1} \\
& \quad+e_{o q} \int_{V_{1}} \int_{V_{11}} G_{i m, j}\left(\vec{r}, \vec{r}_{1}\right)\left(C_{m n k l}^{\prime}\left(\vec{r}_{1}\right)\right. \\
& \cdot G_{k f, l}\left(\vec{r}_{1}, \vec{r}_{11}\right) \\
& \left.\cdot\left(C_{f s o q}^{\prime}\left(\vec{r}_{11}\right)\right)_{, 11 s}\right)_{, 1 n} d V_{11} d V_{1} .
\end{aligned}
$$

The first term in expression (22) is the solution of the problem in the first approximation.

Integrals in (21) and (22) as well as expressions for the moments $\left\langle\varepsilon^{\prime}{ }_{k l}(\vec{r}) \varepsilon^{\prime}{ }_{\phi h}(\vec{r})\right\rangle,\left\langle\lambda^{\prime}(\vec{r}) \varepsilon^{\prime}{ }_{k l}(\vec{r})\right\rangle$, $\left\langle\lambda^{\prime}(\vec{r}) \varepsilon_{\phi h}^{\prime}(\vec{r}) \varepsilon^{\prime}{ }_{k l}(\vec{r})\right\rangle$ can be converted to the second derivative of Green's function using Stieltjes convolution. The Stieltjes convolution of the two functions $f(x)$ and $g(x)$ over the interval $(a, b)$ is defined as function $\Psi(t)$ :

$$
\begin{aligned}
\Psi(t) & \equiv \int_{a}^{b} f(t-x) d g(x) \equiv \int_{a}^{b} g(t-x) d f(x) \\
\Psi(t) & \equiv \int_{a}^{b} f(t-x) g^{\prime}(x) d x \\
& \equiv \int_{a}^{b} g(t-x) f^{\prime}(x) d x .
\end{aligned}
$$

Application of this definition to the integrals containing the first derivative of Green's function and the derivative of the indicator function in the solutions of the boundary value problem (22) gives the next form of the integrals:

$$
\begin{aligned}
& \int_{V_{1}} G_{i m, j}\left(\vec{r}, \vec{r}_{1}\right)\left(C_{m n k l}^{\prime}\left(\vec{r}_{1}\right)\right)_{, 1 n} d V_{1} \\
& =\int_{V_{1}} G_{i m, j n}\left(\vec{r}, \vec{r}_{1}\right) C_{m n k l}^{\prime}\left(\vec{r}_{1}\right) d V_{1} \\
& \int_{V_{1}} \int_{V_{11}} G_{i m, j}\left(\vec{r}, \vec{r}_{1}\right)\left(C_{m n k l}^{\prime}\left(\vec{r}_{1}\right) G_{k f, l}\left(\vec{r}_{1}, \vec{r}_{11}\right)\right. \\
& \left.\cdot\left(C_{m n k l}^{\prime}\left(\vec{r}_{1}\right)\right)_{, 11 s}\right)_{, 1 n} d V_{11} d V_{1} \\
& =\int_{V_{1}} \int_{V_{11}} G_{i m, j n}\left(\vec{r}_{,} \vec{r}_{1}\right) G_{k f, l s}\left(\vec{r}_{1}, \vec{r}_{11}\right) \\
& \cdot C_{m n k l}^{\prime}\left(\vec{r}_{1}\right) C_{m n k l}^{\prime}\left(\vec{r}_{1}\right) d V_{11} d V_{1} .
\end{aligned}
$$

For a two-phase inhomogeneous material, the solution using the second derivatives of the Green's functions takes the form

$$
\begin{aligned}
& u_{i, j}^{\prime(2)}(\vec{r})=e_{k l} \bar{C}_{m n k l} \int_{V_{1}} G_{i m, j n}\left(\vec{r}, \vec{r}_{1}\right) \lambda^{\prime}\left(\vec{r}_{1}\right) d V_{1} \\
& +e_{o q} \bar{C}_{m n k l} \bar{C}_{f s o q} \int_{V_{1}} \int_{V_{11}} G_{i m, j n}\left(\vec{r}, \vec{r}_{1}\right) \\
& \cdot G_{k f, l s}\left(\vec{r}_{1}, \vec{r}_{11}\right) \lambda^{\prime}\left(\vec{r}_{1}\right) \lambda^{\prime}\left(\vec{r}_{11}\right) d V_{11} d V_{1} .
\end{aligned}
$$


The product of the fluctuations of the indicator function $\lambda^{\prime}\left(\vec{r}_{1}\right) \lambda^{\prime}\left(\vec{r}_{11}\right)$ is the correlation function $K_{\lambda}^{(2)}\left(\vec{r}, \vec{r}_{1}\right)$, which characterizes the relative position of the components in microstructure.

By definition, Green's function $G_{k f}\left(\vec{r}, \vec{r}_{1}\right)$ satisfying the expression (15) has a singularity at points $\left|\vec{r}-\vec{r}_{1}\right|=0$. To apply numerical integration methods, the second derivative of Green's function can be represented as the sum of the singular and formal components $G_{k f, l s}\left(\vec{r}, \vec{r}_{1}\right)=G_{k f, l s}^{(s)}\left(\vec{r}, \vec{r}_{1}\right)+$ $G_{k f, l s}^{(f)}\left(\vec{r}, \vec{r}_{1}\right)$.

Then the integrals containing Green's function can be decomposed into two terms [16]:

$$
\begin{aligned}
& \int_{V} G_{k f, l s}\left(\vec{r}, \vec{r}_{1}\right) K_{\lambda}^{(2)}\left(\vec{r}, \vec{r}_{1}\right) d V \\
& =\int_{V_{0}} G_{k f, l s}^{(s)}\left(\vec{r}, \vec{r}_{1}\right) K_{\lambda}^{(2)}\left(\vec{r}, \vec{r}_{1}\right) d V \\
& \quad+\int_{V-V_{0}} G_{k f, l s}^{(f)}\left(\vec{r}_{,} \vec{r}_{1}\right) K_{\lambda}^{(2)}\left(\vec{r}, \vec{r}_{1}\right) d V,
\end{aligned}
$$

where $V_{0}$ is the singularity region of the second derivative of Green's function.

For an isotropic tensor $\left\langle C_{i j k l}\right\rangle$ and in case when the elements of a representative volume are much smaller than the characteristic dimensions of the structure, Green's function for an infinite medium can be used $[1,20]$ :

$$
G_{k n}\left(\vec{r}, \vec{r}_{1}\right)=A \frac{\delta_{k n}}{\left|\vec{r}-\vec{r}_{1}\right|}+B \frac{\left(r_{k}-r_{1 k}\right)\left(r_{n}-r_{1 n}\right)}{\left|\vec{r}-\vec{r}_{1}\right|^{3}}
$$

where $r_{k}$ denotes the $k$ th component of the radius vector $\vec{r}$ and the coefficients $A$ and $B$ are linked with the effective Lame constants $\langle\lambda\rangle$ and $\langle\mu\rangle$ by the relations

$$
A=\frac{\langle\lambda\rangle+\langle 3 \mu\rangle}{8 \pi\langle\mu\rangle\langle\lambda+2 \mu\rangle},
$$

$$
B=\frac{\langle\lambda\rangle+\langle\mu\rangle}{8 \pi\langle\mu\rangle\langle\lambda+2 \mu\rangle} .
$$

Here, $\langle\lambda\rangle=\lambda_{I} p+\lambda_{M}(1-p),\langle\mu\rangle=\mu_{I} p+\mu_{M}(1-p)$.

Expressions representing the singular and formal components for the second derivative of the tensor (27) were obtained in [16]

$$
\begin{aligned}
& G_{k f, l s}^{(s)}\left(\vec{r}, \vec{r}_{1}\right)=-\frac{\delta\left(\vec{r}-\vec{r}_{1}\right)}{3\langle\mu\rangle}\left(\delta_{k f} \delta_{l s}-\frac{1}{5} \kappa \delta_{k f l s}\right) \\
& \quad=\delta\left(\vec{r}-\vec{r}_{1}\right) g_{k f l s} \\
& G_{k f, l s}^{(f)}\left(\vec{r}, \vec{r}_{1}\right)=\frac{1}{8 \pi\langle\mu\rangle\left|\vec{r}-\vec{r}_{1}\right|^{3}}((2-\kappa) \\
& \quad \delta_{k f}\left(3 n_{f} n_{s}-\delta_{f s}\right)-\kappa\left(3 \left(n_{k f} n_{l s}+n_{k l} n_{f s}+n_{k s} n_{f l}\right.\right. \\
& \left.\left.\left.\quad+n_{f s} n_{k l}+n_{f l} n_{k s}\right)-2 I_{k f l s}-15 n_{k} n_{f} n_{l} n_{s}\right)\right),
\end{aligned}
$$

where $\kappa=\langle\lambda+\mu\rangle /\langle\lambda+2 \mu\rangle, n_{i}=\left(r_{i}-r_{1 i}\right) /\left|\vec{r}-\vec{r}_{1}\right|, n_{i j}=n_{i} n_{j}$, $I_{k f l s}=(1 / 2)\left(\delta_{k l} \delta_{f s}+\delta_{k s} \delta_{f l}\right)$.

Taking into account the definition of the generalized Dirac function $\int \delta\left(\vec{r}-\vec{r}_{1}\right) f\left(\vec{r}_{1}\right) d \vec{r}_{1}=f(\vec{r})$, the factor $\delta\left(\vec{r}-\vec{r}_{1}\right)$ in the singular component of the second derivative of Green's tensor leads to a transition from an integral to a constant value; that is, the integral operator $G_{k f, l s}^{(f)}\left(\vec{r}, \vec{r}_{1}\right)$ becomes a constant tensor:

$$
\begin{aligned}
& \int_{V} G_{k f, l s}\left(\vec{r}, \vec{r}_{1}\right) K_{\lambda}^{(2)}\left(\vec{r}, \vec{r}_{1}\right) d V \\
& =g_{k f l s} D_{\lambda}^{(2)} \\
& \quad+\int_{V-V_{0}} G_{k f, l s}^{(f)}\left(\vec{r}, \vec{r}_{1}\right) K_{\lambda}^{(2)}\left(\vec{r}, \vec{r}_{1}\right) d V
\end{aligned}
$$

The moments $\left\langle\varepsilon^{\prime}{ }_{k l}(\vec{r}) \varepsilon^{\prime}{ }_{\phi h}(\vec{r})\right\rangle, \quad\left\langle\lambda^{\prime}(\vec{r}) \varepsilon^{\prime}{ }_{k l}(\vec{r})\right\rangle$, $\left\langle\lambda^{\prime}(\vec{r}) \varepsilon_{\phi h}^{\prime}(\vec{r}) \varepsilon_{k l}^{\prime}(\vec{r})\right\rangle$ can be written in the second derivatives of Green's function, taking into account the expansion (30). The integrals containing the Green functions and the moment functions here and below will be written in abbreviated form as follows:

$$
\begin{aligned}
& I_{1,2,11,21}^{\left(\vec{r}, \vec{r}_{1}, \vec{r}_{2}, \vec{r}_{11}, \vec{r}_{21}\right)} \\
& =D_{\lambda}^{(5)} \int_{V_{1}} \int_{V_{2}} \int_{V_{11}} \int_{V_{21}}\left(G_{i m, j n}^{(f)}\left(\vec{r}, \vec{r}_{1}\right)+G_{j m, i n}^{(f)}\left(\vec{r}_{,} \vec{r}_{1}\right)\right)\left(G_{\alpha \gamma, \beta \eta}^{(f)}\left(\vec{r}_{,} \vec{r}_{2}\right)+G_{\beta \gamma, \alpha \eta}^{(f)}\left(\vec{r}_{,}, \vec{r}_{2}\right)\right) \\
& \times G_{k f, l s}^{(f)}\left(\vec{r}_{x}, \vec{r}_{11}\right) G_{\phi v, h w}^{(f)}\left(\vec{r}_{x}, \vec{r}_{21}\right) f_{\lambda}^{(5)}\left(\vec{r}_{,}, \vec{r}_{1}, \vec{r}_{2}, \vec{r}_{11}, \vec{r}_{21}\right) d V_{21} d V_{11} d V_{2} d V_{1} .
\end{aligned}
$$

Here the subscripts in parentheses denote the presence of the corresponding factors containing Green's function in the integral and the upper indices determine the order and argument of the correlation function. The index $x$ of the 
radius vector is uniquely determined by the integrands and the Dirac convolution rules. The multiplicity of the integral is determined by the number of independent variables $\vec{r}_{i}$. The absence of variables $\vec{r}_{i}$ in brackets means that there is no correlation function in the integrand.

Then, in the second approximation of the problem solution, the mixed moments are written in the following form:

$$
\begin{aligned}
\left\langle\varepsilon_{i j}^{\prime}(\vec{r}) \varepsilon_{\alpha \beta}^{\prime}(\vec{r})\right\rangle & =\frac{1}{4}\left(A_{1}+2 A_{2}+A_{4}\right) \\
\left\langle\lambda^{\prime}(\vec{r}) \varepsilon_{i j}^{\prime}(\vec{r})\right\rangle & =\frac{1}{2}\left(B_{1}+B_{2}\right) \\
\left\langle\lambda^{\prime}(\vec{r}) \varepsilon_{i j}^{\prime}(\vec{r}) \varepsilon_{\alpha \beta}^{\prime}(\vec{r})\right\rangle^{(2)} & =\frac{1}{4}\left(C_{1}+2 C_{2}+C_{4}\right) .
\end{aligned}
$$

The terms $A_{2}, A_{4}, B_{2}, C_{2}, C_{4}$ in these expressions are an additive introduced by the second approximation that refines the solution in the first approximation.

Taking into account the second derivative of Green's function and the expansion into the singular and formal components, the terms of the sought moments have the following form:

$$
\begin{aligned}
& A_{1}=\bar{C}_{m n k l} \bar{C}_{\gamma \eta \phi h} e_{k l} e_{\phi h}\left(I_{1,2}^{\left(\vec{r}_{1}, \vec{r}_{2}\right)}+\left(g_{i j m n}+g_{j m i n}\right)\right. \\
& \cdot I_{2}^{\left(\vec{r}, \vec{r}_{2}\right)}+\left(g_{\alpha \gamma \beta \eta}+g_{\beta \gamma \alpha \eta}\right) I_{1}^{\left(\vec{r}, \vec{r}_{1}\right)}+\left(g_{i j m n}\right. \\
& \left.\left.+g_{\text {jmin }}\right)\left(g_{\alpha \gamma \beta \eta}+g_{\beta \gamma \alpha \eta}\right) D_{\lambda}^{(2)}\right) \\
& A_{2}=e_{k l} e_{b d} \bar{C}_{m n k l} \bar{C}_{\gamma \eta \phi h} \bar{C}_{v w b d}\left(I_{1,2,21}^{\left(\vec{r}_{1}, \vec{r}_{2}, \vec{r}_{21}\right)}+\left(g_{\text {imjn }}\right.\right. \\
& \left.+g_{j \min }\right) I_{2,21}^{\left(\vec{r}, \vec{r}_{2}, \vec{r}_{21}\right)}+\left(g_{\alpha \gamma \beta \eta}+g_{\beta \gamma \alpha \eta}\right) I_{1,21}^{\left(\vec{r}, \vec{r}_{1}, \vec{r}_{21}\right)} \\
& +g_{\phi v h w} I_{1,2}^{\left(\vec{r}, \vec{r}_{1}, \vec{r}_{2}\right)}+\left(g_{\alpha \gamma \beta \eta}+g_{\beta \gamma \alpha \eta}\right) g_{\phi v h w}(1-2 p) \\
& \cdot I_{1}^{\left(\vec{r}, \vec{r}_{1}\right)}+\left(g_{i m j n}+g_{j \min }\right) g_{\phi v h w}(1-2 p) I_{2}^{\left(\vec{r}, \vec{r}_{2}\right)} \\
& +\left(g_{i m j n}+g_{j \min }\right)\left(g_{\alpha \gamma \beta \eta}+g_{\beta \gamma \alpha \eta}\right)(1-2 p) I_{21}^{\left(\vec{r}, \vec{r}_{21}\right)} \\
& \left.+\left(g_{\text {imjn }}+g_{\text {jmin }}\right)\left(g_{\alpha \gamma \beta \eta}+g_{\beta \gamma \alpha \eta}\right) g_{g v h w} D_{\lambda}^{(3)}\right) \\
& A_{4}=e_{o q} e_{b d} \bar{C}_{m n k l} \bar{C}_{\gamma \eta \phi h} \bar{C}_{f s o q} \bar{C}_{r w b d}\left(\left(g_{i m j n}+g_{j m i n}\right)\right. \\
& \cdot\left(g_{\alpha \gamma \beta \eta}+g_{\beta \gamma \alpha \eta}\right) g_{k f l s} g_{\phi v h w} D_{\lambda}^{(4)}+\left(g_{i m j n}+g_{j \min }\right) \\
& \cdot\left(g_{\alpha \gamma \beta \eta}+g_{\beta \gamma \alpha \eta}\right) g_{k f l s}\left(1-3 D_{\lambda}^{(2)}\right) I_{21}^{\left(\vec{r}, \vec{r}_{21}\right)}+\left(g_{i m j n}\right. \\
& \left.+g_{\text {jmin }}\right) g_{k f l s} g_{\phi v h w}\left(1-3 D_{\lambda}^{(2)}\right) I_{2}^{\left(\vec{r}, \vec{r}_{2}\right)}+\left(g_{\alpha \gamma \beta \eta}\right. \\
& \left.+g_{\beta \gamma \alpha \eta}\right) g_{k f l s} g_{\phi v h w}\left(1-3 D_{\lambda}^{(2)}\right) I_{1}^{\left(\vec{r}, \vec{r}_{1}\right)}+\left(g_{i m j n}\right. \\
& \left.+g_{\text {jmin }}\right)\left(g_{\alpha \gamma \beta \eta}+g_{\beta \gamma \alpha \eta}\right)\left[(1-2 p) I_{11,21}^{\left(\vec{r}, \vec{r}_{11}, \vec{r}_{21}\right)}\right.
\end{aligned}
$$

$$
\begin{aligned}
& \left.+D_{\lambda}^{(2)} I_{11,21}^{\left(\vec{r}_{11}, \vec{r}_{21}\right)}\right]+\left(g_{\text {imjn }}+g_{\text {jmin }}\right) \\
& \cdot g_{k f l s}\left[(1-2 p) I_{2,21}^{\left(\vec{r}, \vec{r}_{2}, \vec{r}_{21}\right)}+D_{\lambda}^{(2)} I_{2,21}^{\left(\vec{r}_{2}, \vec{r}_{21}\right)}\right] \\
& +\left(g_{i m j n}+g_{j m i n}\right) g_{\phi v h w}\left[(1-2 p) I_{2,11}^{\left(\vec{r}, \vec{r}_{2}, \vec{r}_{11}\right)}\right. \\
& \left.+D_{\lambda}^{(2)} I_{2,11}^{\left(\vec{r}_{2}, \vec{r}_{11}\right)}\right]+\left(g_{\alpha \gamma \beta \eta}+g_{\beta \gamma \alpha \eta}\right) \\
& \cdot g_{k f l s}\left[(1-2 p) I_{1,21}^{\left(\vec{r}, \vec{r}_{1}, \vec{r}_{21}\right)}+D_{\lambda}^{(2)} I_{1,21}^{\left(\vec{r}_{1}, \vec{r}_{21}\right)}\right] \\
& +\left(g_{\alpha \gamma \beta \eta}+g_{\beta \gamma \alpha \eta}\right) g_{\phi v h w}\left[(1-2 p) I_{1,11}^{\left(\vec{r}, \vec{r}_{1}, \vec{r}_{11}\right)}\right. \\
& \left.+D_{\lambda}^{(2)} I_{1,11}^{\left(\vec{r}_{1}, \vec{r}_{11}\right)}\right]+g_{k f l s} g_{\phi v h w}\left[(1-2 p) I_{1,2}^{\left(\vec{r}, \vec{r}_{1}, \vec{r}_{2}\right)}\right. \\
& \left.+D_{\lambda}^{(2)} I_{1,2}^{\left(\vec{r}_{1}, \vec{r}_{2}\right)}\right]+\left(g_{\text {imjn }}+g_{\text {jmin }}\right) I_{2,11,21}^{\left(\vec{r}, \vec{r}_{2}, \vec{r}_{11}, \vec{r}_{21}\right)} \\
& +\left(g_{\alpha \gamma \beta \eta}+g_{\beta \gamma \alpha \eta}\right) I_{1,11,21}^{\left(\vec{r}, \vec{r}_{1}, \vec{r}_{11}, \vec{r}_{21}\right)} \\
& +g_{k f l s} I_{1,2,21}^{\left(\vec{r}, \vec{H}_{1}, \vec{r}_{2}, \vec{r}_{21}\right)}+g_{\phi v h w} I_{1,2,11}^{\left(\vec{r}, \vec{r}_{1}, \vec{r}_{2}, \vec{r}_{11}\right)} \\
& \left.+I_{1,2,11,21}^{\left(\vec{r}_{1}, \vec{r}_{2}, \vec{r}_{11}, \vec{r}_{21}\right)}\right) \\
& B_{1}=e_{k l} \bar{C}_{m n k l}\left[\left(g_{i m j n}+g_{j m i n}\right) D_{\lambda}^{(2)}+I_{1}^{\left(\vec{r}, \vec{r}_{1}\right)}\right] \\
& B_{2}=e_{o q} \bar{C}_{m n k l} \bar{C}_{f s o q}\left(\left(g_{i m j n}+g_{j m i n}\right) g_{k f l s} D_{\lambda}^{(3)}\right. \\
& +\left(g_{i m j n}+g_{\text {jmin }}\right)(1-2 p) I_{11}^{\left(\vec{r}, \vec{r}_{11}\right)}+g_{k f l s}(1-2 p) \\
& \left.\cdot I_{1}^{\left(\vec{r}, \vec{r}_{1}\right)}+I_{1,11}^{\left(\vec{r}, \vec{r}_{1}, \vec{r}_{11}\right)}\right) \\
& C_{1}=e_{k l} e_{\phi h} \bar{C}_{m n k l} \bar{C}_{\gamma \eta \phi h}\left(I_{1,2}^{\left(\vec{r}, \vec{r}_{1}, \vec{r}_{2}\right)}+\left(g_{i m j n}+g_{j m i n}\right)\right. \\
& \cdot(1-2 p) I_{2}^{\left(\vec{r}, \vec{r}_{2}\right)}+\left(g_{\alpha \gamma \beta \eta}+g_{\beta \gamma \alpha \eta}\right)(1-2 p) I_{1}^{\left(\vec{r}, \vec{r}_{1}\right)} \\
& \left.+\left(g_{\text {imjn }}+g_{\text {jmin }}\right)\left(g_{\alpha \gamma \beta \eta}+g_{\beta \gamma \alpha \eta}\right) D_{\lambda}^{(2)}\right) \\
& C_{2}=e_{k l} e_{b d} \bar{C}_{m n k l} \bar{C}_{\gamma \eta \phi h} \bar{C}_{v w b d}\left(( g _ { i m j n } + g _ { j m i n } ) \left(g_{\alpha \gamma \beta \eta}\right.\right. \\
& \left.+g_{\beta \gamma \alpha \eta}\right) g_{\phi v h w} D_{\lambda}^{(4)}+\left(g_{i m j n}+g_{j m i n}\right)\left(g_{\alpha \gamma \beta \eta}\right. \\
& \left.+g_{\beta \gamma \alpha \eta}\right)\left(1-3 D_{\lambda}^{(2)}\right) I_{21}^{\left(\vec{r}, \vec{r}_{21}\right)}+\left(g_{\text {imjn }}+g_{j \text { min }}\right) \\
& \cdot g_{\phi v h w}\left(1-3 D_{\lambda}^{(2)}\right) I_{2}^{\left(\vec{r}, \vec{r}_{2}\right)}+\left(g_{\alpha \gamma \beta \eta}+g_{\beta \gamma \alpha \eta}\right) \\
& \cdot g_{\phi v h w}\left(1-3 D_{\lambda}^{(2)}\right) I_{1}^{\left(\vec{r}, \vec{r}_{1}\right)}+\left(g_{\text {imjn }}+g_{\text {jmin }}\right) \\
& \cdot\left[(1-2 p) I_{2,21}^{\left(\vec{r}, \vec{r}_{2}, \vec{r}_{21}\right)}+D_{\lambda}^{(2)} I_{2,21}^{\left(\vec{r}_{2}, \vec{r}_{21}\right)}\right]+\left(g_{\alpha \gamma \beta \eta}\right. \\
& \left.+g_{\beta \gamma \alpha \eta}\right)\left[(1-2 p) I_{1,21}^{\left(\vec{r}, \vec{r}_{1}, \vec{r}_{21}\right)}+D_{\lambda}^{(2)} I_{1,21}^{\left(\vec{r}_{1}, \vec{r}_{21}\right)}\right] \\
& +g_{\phi v h w}\left[(1-2 e) I_{1,2}^{\left(\vec{r}, \vec{r}_{1}, \vec{r}_{2}\right)}+D_{\lambda}^{(2)} I_{1,2}^{\left(\vec{r}_{1}, \vec{r}_{2}\right)}\right]
\end{aligned}
$$




$$
\begin{aligned}
& \left.+I_{1,2,21}^{\left(\vec{r}, \vec{r}_{1}, \vec{r}_{2}, \vec{r}_{21}\right)}\right) \\
& C_{4}=e_{o q} e_{b d} \bar{C}_{m n k l} \bar{C}_{\gamma \eta \phi h} \bar{C}_{f s o q} \bar{C}_{v w b d}\left(\left(g_{i m j n}+g_{j m i n}\right)\right. \\
& \cdot\left(g_{\alpha \gamma \beta \eta}+g_{\beta \gamma \alpha \eta}\right) g_{k f l s} g_{\phi v h w} D_{\lambda}^{(5)}+\left(g_{i m j n}+g_{j m i n}\right) \\
& \cdot\left(g_{\alpha \gamma \beta \eta}+g_{\beta \gamma \alpha \eta}\right) g_{k f l s}\left(p^{-1} D_{\lambda}^{(4)}-p^{3}\right) I_{21}^{\left(\vec{r}, \vec{r}_{21}\right)} \\
& +\left(g_{\text {imjn }}+g_{\text {jmin }}\right)\left(g_{\alpha \gamma \beta \eta}+g_{\beta \gamma \alpha \eta}\right) g_{\phi v h w}\left(p^{-1} D_{\lambda}^{(4)}\right. \\
& \left.-p^{3}\right) I_{11}^{\left(\vec{r}, \vec{r}_{11}\right)}+\left(g_{i m j n}+g_{j m i n}\right) g_{k f l s} g_{\phi v h w}\left(p^{-1} D_{\lambda}^{(4)}\right. \\
& \left.-p^{3}\right) I_{2}^{\left(\vec{r}, \vec{r}_{2}\right)}+\left(g_{\alpha \gamma \beta \eta}+g_{\beta \gamma \alpha \eta}\right) g_{k f l s} g_{\phi v h w}\left(p^{-1} D_{\lambda}^{(4)}\right. \\
& \left.-p^{3}\right) I_{1}^{\left(\vec{r}, \vec{r}_{1}\right)}+\left(g_{\text {imjn }}+g_{\text {jmin }}\right)\left(g_{\alpha \gamma \beta \eta}+g_{\beta \gamma \alpha \eta}\right) \\
& \cdot\left[\left(1-3 D_{\lambda}^{(2)}\right) I_{11,21}^{\left(\vec{r}, \vec{r}_{11}, \vec{r}_{21}\right)}+D_{\lambda}^{(3)} I_{11,21}^{\left(\vec{r}_{11}, \vec{r}_{21}\right)}\right] \\
& +\left(g_{\text {imjn }}+g_{\text {jmin }}\right) g_{k f l s}\left[\left(1-3 D_{\lambda}^{(2)}\right) I_{2,21}^{\left(\vec{r}, \vec{r}_{2}, \vec{r}_{21}\right)}\right. \\
& \left.+D_{\lambda}^{(3)} I_{2,21}^{\left(\vec{r}_{2}, \vec{r}_{21}\right)}\right]+\left(g_{\text {imjn }}+g_{\text {jmin }}\right) \\
& \cdot g_{\phi v h w}\left[\left(1-3 D_{\lambda}^{(2)}\right) I_{2,11}^{\left(\vec{r}, \vec{r}_{2}, \vec{r}_{11}\right)}+D_{\lambda}^{(3)} I_{2,11}^{\left(\vec{r}_{2}, \vec{r}_{11}\right)}\right] \\
& +\left(g_{\alpha \gamma \beta \eta}+g_{\beta \gamma \alpha \eta}\right) g_{k f l s}\left[\left(1-3 D_{\lambda}^{(2)}\right) I_{1,21}^{\left(\vec{r}, \vec{r}_{1}, \vec{r}_{21}\right)}\right. \\
& \left.+D_{\lambda}^{(3)} I_{1,21}^{\left(\vec{r}_{1}, \vec{r}_{21}\right)}\right]+\left(g_{\alpha \gamma \beta \eta}+g_{\beta \gamma \alpha \eta}\right) \\
& \cdot g_{\phi v h w}\left[\left(1-3 D_{\lambda}^{(2)}\right) I_{1,11}^{\left(\vec{r}, \vec{r}_{1}, \vec{r}_{11}\right)}+D_{\lambda}^{(3)} I_{1,11}^{\left(\vec{r}_{1}, \vec{r}_{11}\right)}\right] \\
& +g_{k f l s} g_{\phi v h w}\left[\left(1-3 D_{\lambda}^{(2)}\right) I_{1,2}^{\left(\vec{r}, \vec{r}_{1}, \vec{r}_{2}\right)}\right. \\
& \left.+D_{\lambda}^{(3)} I_{1,2}^{\left(\vec{r}_{1}, \vec{r}_{2}\right)}\right]+\left(g_{\text {imjn }}+g_{\text {jmin }}\right) \\
& \cdot\left[(1-2 p) I_{2,11,21}^{\left(\vec{r}, \vec{r}_{2}, \vec{r}_{11}, \vec{r}_{21}\right)}+D_{\lambda}^{(2)} I_{2,11,21}^{\left(\vec{r}_{2}, \vec{r}_{11}, \vec{r}_{21}\right)}\right. \\
& \left.+p^{3} D_{\lambda}^{(2)} I_{2,11,21}\right]+\left(g_{\alpha \gamma \beta \eta}+g_{\beta \gamma \alpha \eta}\right) \\
& \cdot\left[(1-2 p) I_{1,11,21}^{\left(\vec{r}, \vec{r}_{1}, \vec{r}_{11}, \vec{r}_{21}\right)}+D_{\lambda}^{(2)} I_{1,11,21}^{\left(\vec{r}_{1}, \vec{r}_{11}, \vec{r}_{21}\right)}\right. \\
& \left.+p^{3} D_{\lambda}^{(2)} I_{1,11,21}\right]+g_{k f l s}\left[(1-2 p) I_{1,2,21}^{\left(\vec{r}, \vec{r}_{1}, \vec{r}_{2}, \vec{r}_{21}\right)}\right. \\
& \left.+D_{\lambda}^{(2)} I_{1,2,21}^{\left(\vec{r}_{1}, \vec{r}_{2}, \vec{r}_{21}\right)}+p^{3} D_{\lambda}^{(2)} I_{1,2,21}\right] \\
& +g_{\phi v h w}\left[(1-2 p) I_{1,2,11}^{\left(\vec{r}, \vec{r}_{1}, \vec{r}_{2}, \vec{r}_{11}\right)}+D_{\lambda}^{(2)} I_{1,2,11}^{\left(\vec{r}_{1}, \vec{r}_{2}, \vec{r}_{11}\right)}\right. \\
& \left.\left.+p^{3} D_{\lambda}^{(2)} I_{1,2,11}\right]+I_{1,2,11,21}^{\left(\vec{r}, \vec{r}_{1}, \vec{r}_{2}, \vec{r}_{11}, \vec{r}_{21}\right)}\right) .
\end{aligned}
$$

These expressions explicitly contain the structural correlation functions up to the 5th order and represent a generalized case of expressions for the statistical characteristics of fields. In previously obtained results, the correlation functions of second- and third-order maximum were taken into account based on first-order approximations of the solution of a boundary value problem. For instance, in the two-point approximation only pair interactions are taken into account; therefore correlation functions of higher order than binary ones are excluded $[16,20]$.

Eliminating all the terms containing the formal component from the last expressions, a singular approximation for statistical characteristics is derived [16]. Because of negligibility of terms containing correlation functions, the singular approximation does not take into account the real distribution of components in a representative volume but depends only on the volume fraction:

$$
\begin{aligned}
& \left\langle\varepsilon^{\prime}{ }_{i j}(\vec{r}) \varepsilon_{\alpha \beta}^{\prime}(\vec{r})\right\rangle \\
& =\frac{1}{4}\left(\bar{C}_{m n k l} \bar{C}_{\gamma \eta \phi h} e_{k l} e_{\phi h}\left(g_{i j m n}+g_{j m i n}\right)\right. \\
& \cdot\left(g_{\alpha \gamma \beta \eta}+g_{\beta \gamma \alpha \eta}\right) D_{\lambda}^{(2)} \\
& +2 e_{k l} e_{b d} \bar{C}_{m n k l} \bar{C}_{\gamma \eta \phi h} \bar{C}_{v w b d}\left(g_{i m j n}+g_{j m i n}\right) \\
& \cdot\left(g_{\alpha \gamma \beta \eta}+g_{\beta \gamma \alpha \eta}\right) g_{g v h w} D_{\lambda}^{(3)} \\
& +e_{o q} e_{b d} \bar{C}_{m n k l} \bar{C}_{\gamma \eta \phi h} \bar{C}_{f s o q} \bar{C}_{v w b d}\left(g_{i m j n}+g_{j m i n}\right) \\
& \left.\cdot\left(g_{\alpha \gamma \beta \eta}+g_{\beta \gamma \alpha \eta}\right) g_{k f l s} g_{\phi v h w} D_{\lambda}^{(4)}\right) \\
& \left\langle\lambda^{\prime}(\vec{r}) \varepsilon^{\prime}{ }_{i j}(\vec{r})\right\rangle=\frac{1}{2}\left(e_{k l} \bar{C}_{m n k l}\left(g_{i m j n}+g_{j m i n}\right) D_{\lambda}^{2}\right. \\
& \left.+e_{o q} \bar{C}_{m n k l} \bar{C}_{f s o q}\left(g_{i m j n}+g_{j m i n}\right) g_{k f l s} D_{\lambda}^{(3)}\right) \\
& \left\langle\lambda^{\prime}(\vec{r}) \varepsilon_{i j}^{\prime}(\vec{r}) \varepsilon_{\alpha \beta}^{\prime}(\vec{r})\right\rangle \\
& =\frac{1}{4}\left(e_{k l} e_{\phi h} \bar{C}_{m n k l} \bar{C}_{\gamma \eta \phi h}\left(g_{i m j n}+g_{j m i n}\right)\right. \\
& \cdot\left(g_{\alpha \gamma \beta \eta}+g_{\beta \gamma \alpha \eta}\right) D_{\lambda}^{(2)} \\
& +2 e_{k l} e_{b d} \bar{C}_{m n k l} \bar{C}_{\gamma \eta \phi h} \bar{C}_{v w b d}\left(g_{i m j n}+g_{j m i n}\right) \\
& \cdot\left(g_{\alpha \gamma \beta \eta}+g_{\beta \gamma \alpha \eta}\right) g_{\phi v h w} D_{\lambda}^{(4)} \\
& +e_{o q} e_{b d} \bar{C}_{m n k l} \bar{C}_{\gamma \eta \phi h} \bar{C}_{f s o q} \bar{C}_{v w b d}\left(g_{i m j n}+g_{j m i n}\right) \\
& \left.\cdot\left(g_{\alpha \gamma \beta \eta}+g_{\beta \gamma \alpha \eta}\right) g_{k f l s} g_{\phi v h w} D_{\lambda}^{(5)}\right) \text {. }
\end{aligned}
$$

Unlike the singular approximation, multipoint methods allow to take into account the morphological features of the microstructure of inhomogeneous media by means of a set of correlation functions.

It is obvious that the contribution made by the second approximation of the solution of the SBVP is significant in terms of the complication of analytic expressions; however, it contains terms that do not depend on the correlation functions and which can be neglected during numerical integration due to the insignificance of their contribution to the values of the moments. 
Numerical calculation of the obtained expressions can be simplified by specifying types of loading due to the symmetry properties of the tensors in the expressions. Numerical calculation of the values of the multidimensional integrals can be implemented in the commercial packages, which offer a large variation of integration schemes. Singularity isolation allows integration without additional analysis of integrands. That significantly increases the computational speed. The solution of the obtained integral equations can be effectively parallelized.

Thus, by specifying the elastic characteristics of the components of the composite, the stress-strain state (using the macroscopic strain tensor), knowing the geometry of the structure (the volume fraction, the size of the inclusions) and the analytical expressions for the microstructural correlation functions, the moments of the first- and second-orders of the fields of local strain and stress can be calculated using methods of numerical integration.

Some examples of the numerical implementation of the derived stochastic micromechanical model are presented in $[19,25]$, where calculations for a case study of elastic and elastoplastic composites with different microstructural properties were obtained for various loading conditions of the RVE. It is worth mentioning that contribution of the second approximation of the integral equation (21) is more significant when the correlation functions of 4 th and 5 th order demonstrate more oscillatory behavior. The latter happens when inclusions are unevenly distributed inside the RVE and due to the fact that correlation functions of each subsequent order appeared to be more sensitive to the features of microstructural morphology. Empirically it was established that the third-order functions reflect changes in the form of inclusions, while fourth-order functions describe how inclusions are grouped and how their sizes are distributed. Thus, it was shown that the second approximation term in (22) almost does not make any addition for RVEs with uniformly distributed inclusions (the difference between results of local fields modeling between the first and second solution approximation was around 3\%). However, for more complex structures, when inclusions form clusters, the effect of the microstructure becomes more valuable and is explicitly accounted in the model with help of the high order correlation functions.

\section{Conclusions}

The paper presents the derivation of analytical expressions for the moments of the first and second order of local stress and strain in the components of a two-phase structurally inhomogeneous medium using multipoint correlation functions and second derivatives of Green's functions. The advantage of this method is that it allows to take into account the information about the internal structure of the medium, formalized by multipoint correlation functions, with high precision.

The obtained statistical moments have a number of practical applications. The statistical description of local microstructural fields of stresses and strains is one of the tools for assessing the initiation of damage growth and understanding of nonlinear mechanical phenomena. Based on the statistical metrics, a model for the multiscale failure probability calculation can be implemented. Representation of the failure criteria in a statistical form is required for that, followed by statistical evaluation of the values of the critical quantities. Homogenization methods based on multipoint statistical instruments allow obtaining a fast numerical estimate for effective properties with allowance for microscale interactions.

\section{Conflicts of Interest}

The author declares that they have no conflicts of interest.

\section{Acknowledgments}

This work was supported by the Grant of the President of Russian Federation for state support of young Russian scientists (MK-2395.2017.1) and by the Russian Foundation for Basic Research (Projects 16-41-590259-r_a, 16-01-00327_a).

\section{References}

[1] V. A. Buryachenko, Micromehcanics of Heterogenous Materials, Springer US, Boston, MA, USA, 2007.

[2] S. Torquato, Random Heterogeneous Materials, Springer, New York, NY, USA, 2002.

[3] E. Kroener, "Statistical Modelling," in Model. Small Deform. Polycrystals, Springer, Dordrecht, The Netherlands, 1986.

[4] M. Beran, "Statistical Continuum Theories," Journal of Rheology, vol. 9, no. 1, pp. 339-355, 1965.

[5] V. A. Buryachenko and F. G. Rammerstorfer, "Thermoelastic stress fluctuations in random-structure coated particulate composites," European Journal of Mechanics - A/Solids, vol. 17, no. 5, pp. 763-788, 1998.

[6] A. G. Evans, "Microfracture from thermal expansion anisotropy-I. Single phase systems," Acta Metallurgica et Materialia, vol. 26, no. 12, pp. 1845-1853, 1978.

[7] Y. Fu and A. G. Evans, "Some effects of microcracks on the mechanical properties of brittle solids-I. Stress, strain relations," Acta Metallurgica et Materialia, vol. 33, no. 8, pp. 1515-1523, 1985.

[8] V. Tvergaard and J. W. Hutchinson, "Microcracking in Ceramics Induced by Thermal Expansion or Elastic Anisotropy," Journal of the American Ceramic Society, vol. 71, no. 3, pp. 157-166, 1988.

[9] Z. Hashin, "Assessment of the Self Consistent Scheme Approximation: Conductivity of Particulate Composites," Journal of Composite Materials, vol. 2, no. 3, pp. 284-300, 1968.

[10] R. W. Zimmerman, "Effective conductivity of a twodimensional medium containing elliptical inhomogeneities," Proceedings of the Royal Society A Mathematical, Physical and Engineering Sciences, vol. 452, no. 1950, pp. 1713-1727, 1996.

[11] G. W. Milton, "The coherent potential approximation is a realizable effective medium scheme," Communications in Mathematical Physics, vol. 99, no. 4, pp. 463-500, 1985.

[12] L. Weber, C. Fischer, and A. Mortensen, "On the influence of the shape of randomly oriented, non-conducting inclusions in a conducting matrix on the effective electrical conductivity," Acta Materialia, vol. 51, no. 2, pp. 495-505, 2003. 
[13] C. H. Chen and Y. C. Wang, "Effective thermal conductivity of misoriented short-fiber reinforced thermoplastics," Mechanics of Materials, vol. 23, no. 3, pp. 217-228, 1996.

[14] Y. Benveniste, "On the effective thermal conductivity of multiphase composites," ZAMP Zeitschrift für angewandte Mathematik und Physik, vol. 37, no. 5, pp. 696-713, 1986.

[15] M. I. Shvidler, Statistical Hydrodynamics of Porous Media, Nauka, Moscow, 1985.

[16] T. D. Shermergor, The Theory of Elasticity of Microinhomogeneous Media, Nauka, Moscow, 1977.

[17] L. P. Khoroshun, B. P. Maslov, E. N. Shikula, and L. V. Nazarenko, Statistical Mechanics and Effective Properties of Materials, Naukova Dumka, Kiev, Ukraine, 1993.

[18] M. Tashkinov, V. Wildemann, and N. Mikhailova, "Method of successive approximations in stochastic elastic boundary value problem for structurally heterogenous materials," Computational Materials Science, vol. 52, no. 1, pp. 101-106, 2012.

[19] M. A. Tashkinov, Mechanics of Advanced Materials, Springer International Publishing, 2015, pp. 43-78.

[20] S. D. Volkov and V. P. Stavrov, "Statistical Mechanics of Composite Materials," Belorussian State University, 1978 (Russian).

[21] V. A. Buryachenko, "Solution of general integral equations of micromechanics of heterogeneous materials," International Journal of Solids and Structures, vol. 51, no. 23-24, pp. 3823-3843, 2014.

[22] B. Budiansky, "On the elastic moduli of some heterogeneous materials," Journal of the Mechanics and Physics of Solids, vol. 13, no. 4, pp. 223-227, 1965.

[23] R. Hill, "A self-consistent mechanics of composite materials," Journal of the Mechanics and Physics of Solids, vol. 13, no. 4, pp. 213-222, 1965.

[24] Ş. S. Bayin, Mathematical Methods in Science and Engineering, 2006.

[25] M. Tashkinov, "Micro-scale modeling of phase-level elastic fields of $\mathrm{SiC}$ reinforced metal matrix multiphase composites using statistical approach," Computational Materials Science, vol. 116, pp. 113-121, 2016. 


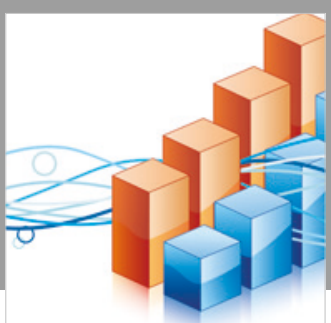

Advances in

Operations Research

\section{-n-m}
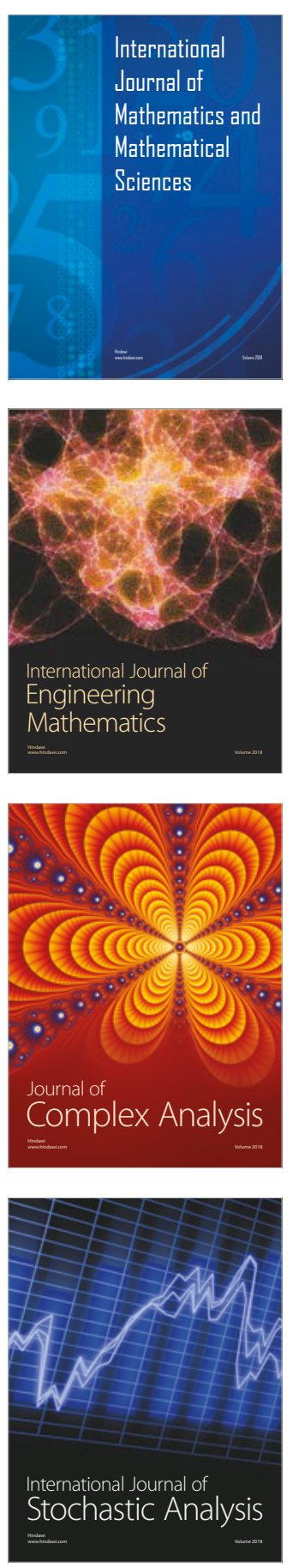
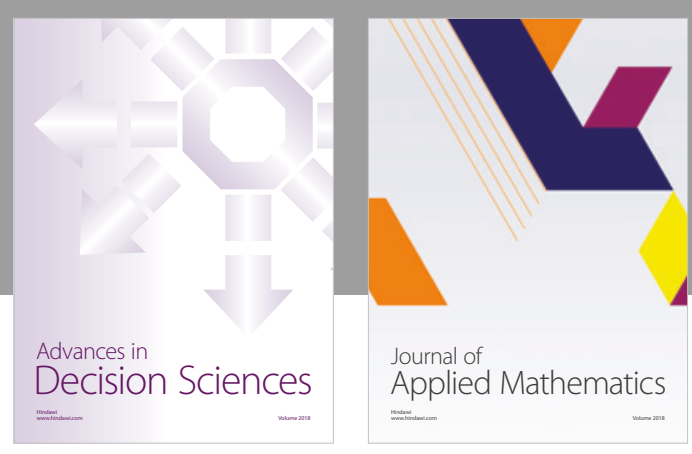

Journal of

Applied Mathematics
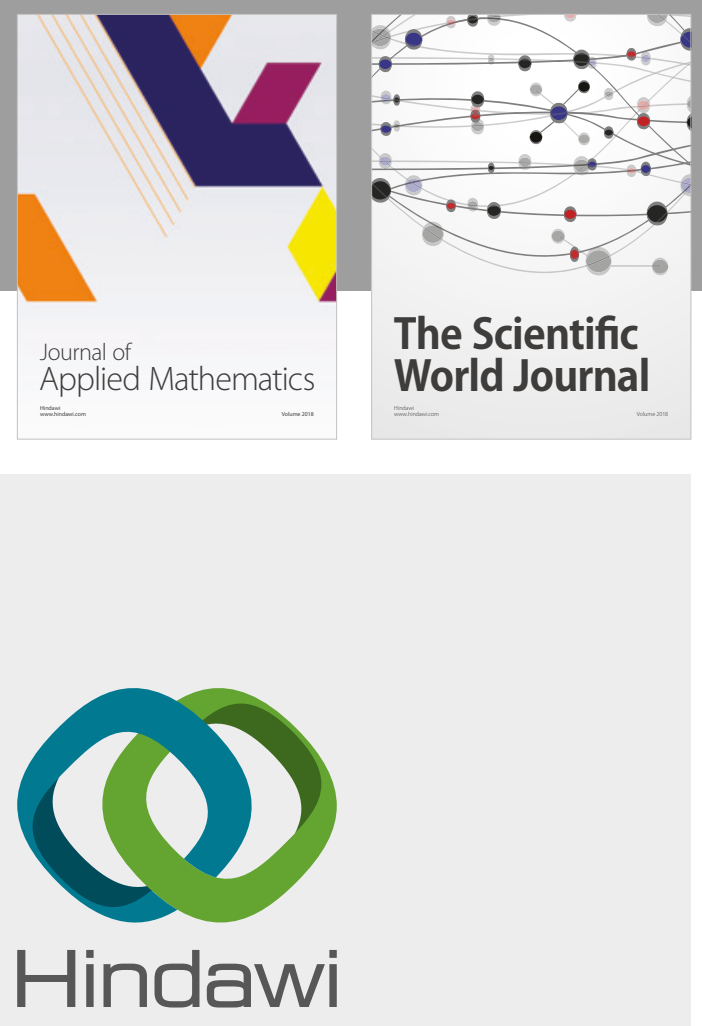

Submit your manuscripts at

www.hindawi.com

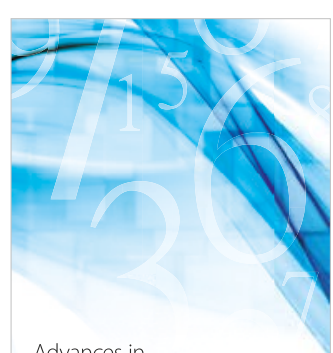

Advances in
Numerical Analysis
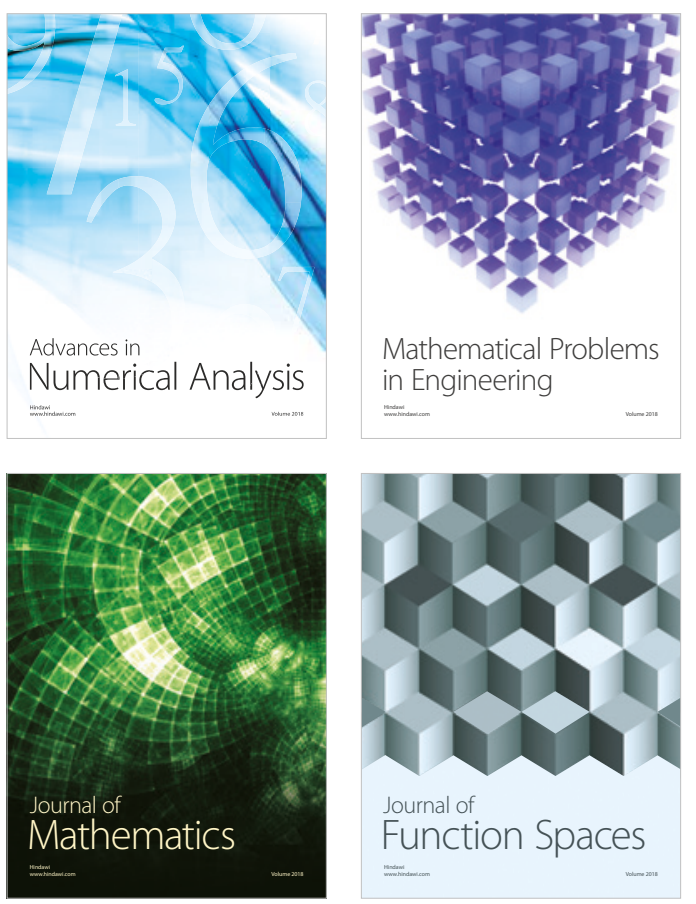

Mathematical Problems in Engineering

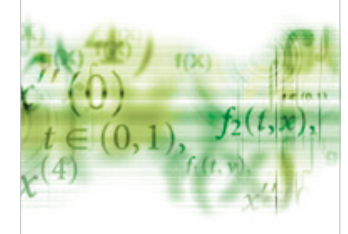

International Journal of

Differential Equations

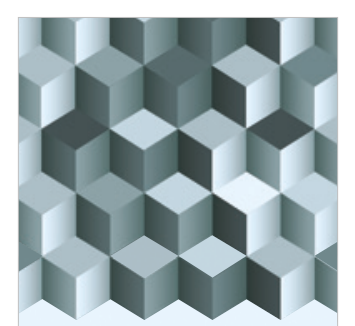

Journal of

Function Spaces

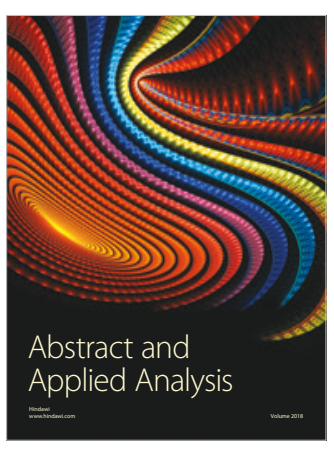

The Scientific

World Journal

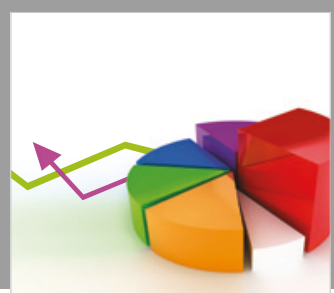

Journal of

Probability and Statistics
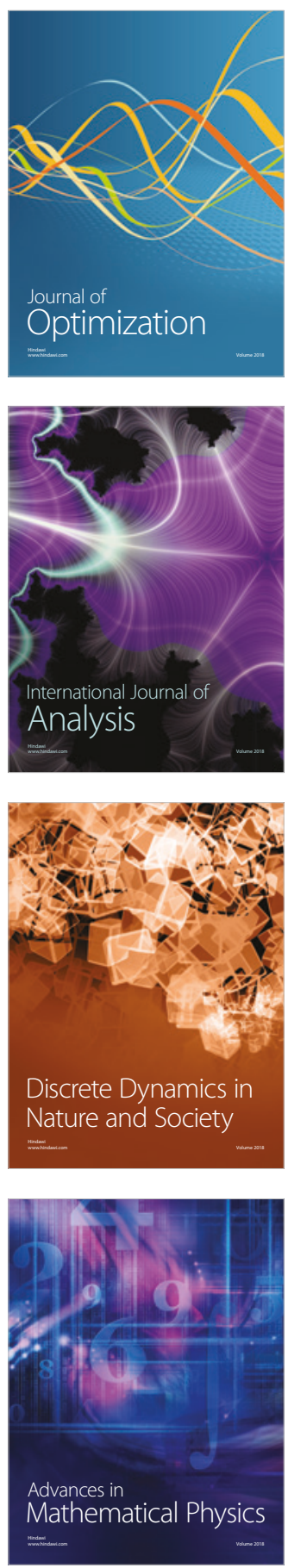